\title{
Mapa conceptual sobre salud pública y propiedad intelectual en Cuba: actualización 2020
}

\author{
Beatriz García Delgado', José Luis Di Fabio² y Jaume Vidal Casanovas ${ }^{3}$
}

Forma de citar

García Delgado B, Di Fabio JL, Vidal Casanovas J. Mapa conceptual sobre salud pública y propiedad intelectual en Cuba: actualización 2020. Rev Panam Salud Publica. 2020;44:e76. https://doi.org/10.26633/RPSP.2020.76

RESUMEN

En este estudio se identifican y describen los cambios introducidos en el entramado constitucional y legal, y la política económica y social de Cuba entre los años 2015 y 2020 que inciden en el acceso a la salud y se actualiza el mapa conceptual sobre salud pública y propiedad intelectual en Cuba (MC SPPIC). Se realizó la búsqueda de documentos, ajustada al período de tiempo analizado, en la Gaceta Oficial de la República de Cuba y los sitios en Internet de la Oficina Cubana de la Propiedad Industrial, los organismos de la Administración Central del Estado y del Grupo de las Industrias Biotecnológica y Farmacéutica BioCubaFarma. El MC SPPIC actualizado refleja los cambios en la nueva Constitución de la República de Cuba adoptada en 2019, la actualización de los Lineamientos de la Política Económica y Social para el quinquenio 2016-2021 y las leyes, decretos leyes y otras disposiciones legales relacionados con la propiedad intelectual en el período 2015-2020. La Política sobre el Sistema de Propiedad Industrial aprobada se fortaleció con legislaciones específicas para la protección de la propiedad industrial, en especial las que inciden en la protección de los resultados obtenidos por la industria biotecnológica y farmacéutica cubana. Los cambios reflejados en el MC SPPIC actualizado favorecen la interacción y la sinergia entre los diversos actores que inciden en el acceso a la salud —en su sentido más amplio— de la población cubana.

Palabras clave Propiedad intelectual; legislación como asunto; industria farmacéutica; derecho a la salud; Cuba.

En el año 2015 se publicó el mapa conceptual sobre salud pública y propiedad intelectual en Cuba (MC SPPIC) (1), que tuvo como antecedentes la Estrategia Global sobre Salud Pública, Innovación y Propiedad Intelectual, contenida en la Resolución WHA61.21 de la Organización Mundial de la Salud (OMS) (2), y la Perspectiva Regional sobre Salud Pública, Innovación y Propiedad Intelectual, contenida en la Resolución CD48.R15 de la Organización Panamericana de la Salud (OPS) (3). Este último documento sirvió de base para que la OPS desarrollara el proyecto Mapa Conceptual sobre Salud Pública y Propiedad Intelectual, dirigido a ofrecer un diagnóstico objetivo de los elementos de la propiedad intelectual relacionados con la salud pública. Ese mapa conceptual, una vez adaptado a la realidad específica de los países, facilitaría la acción de las autoridades nacionales para regular y promover el acceso a las

\footnotetext{
1 Consultora independiente, La Habana, Cuba. $\bowtie$ beatrizgarcia@infomed.sld.cu

2 Consultor independiente, Washington, D.C., Estados Unidos de América.
}

tecnologías sanitarias con la correcta gestión de los derechos de propiedad intelectual, todo dentro de los marcos legales vigentes y las estructuras institucionales existentes.

La OMS y la OPS han aprobado importantes documentos e informes en temas relacionados con la propiedad intelectual y el acceso a la salud (2-4). Por su parte, diversos autores han tratado el tema de los mapas conceptuales y han resaltado su utilidad en la planificación estratégica y el desarrollo de la atención sanitaria (5-7).

El MC SPPIC (1) constituye una herramienta estratégica que permite vincular las fases de planeación, ejecución y evaluación de las intervenciones públicas relacionadas con la salud mediante la visualización de los actores e instituciones involucrados, así como de sus respectivos marcos legales de competencia. En ese proceso se destacan los objetivos comunes, 
que en este caso específico son la consolidación de las capacidades públicas de investigación, desarrollo y producción, y el derecho de la población al acceso pleno a la salud.

Después de la publicación del MC SPPIC, Cuba ha estado inmersa en un proceso de profundas reformas de su entramado constitucional y legal, y se han actualizado los Lineamientos Estratégicos de la Política Económica y Social, documento cardinal de la estrategia de desarrollo del país. Estos cambios inciden de manera directa en la gestión de los derechos de propiedad intelectual, el desarrollo de la industria biotecnológica y farmacéutica, los derechos a la salud y el acceso a ella por la población.

Simultáneamente, se han modificado o aprobado nuevas políticas públicas relacionadas con la esfera de la ciencia y la innovación, y su protección (8).

En Cuba, la Política del Sistema de Propiedad Industrial y las legislaciones concurrentes sirven de fundamento al Sistema de Propiedad Industrial, que constituye el conjunto de relaciones funcionales para la gestión de la propiedad industrial en el país y con otros mercados de interés. Este sistema se fundamenta en los principios de política, normas nacionales, y tratados y acuerdos internacionales suscritos en la materia (9-12). En el cuadro 1 se presentan los principios de la Política del Sistema de Propiedad Industrial y del Sistema de Propiedad Industrial más estrechamente vinculados con la salud.

Los cambios registrados en Cuba, conjuntamente con las medidas adoptadas para proteger la salud pública, salvaguardan el interés general en torno al desarrollo científico-tecnológico y económico-social cubano y de las llamadas empresas de alta tecnología (13-15), vinculadas principalmente a la industria biotecnológica y farmacéutica. Esos cambios han sido de vital importancia para mejorar el acceso de la población cubana a las tecnologías sanitarias y sus resultados.

El objetivo del presente trabajo es identificar y describir los cambios introducidos en el entramado constitucional y legal, y la política económica y social de Cuba entre los años 2015 y 2020 que inciden en el acceso a la salud y, a partir de ellos, actualizar el MC SPPIC.

\section{FUNDAMENTOS Y PROCEDIMIENTOS}

Se partió de la metodología de generación y gestión del conocimiento (16-18), que consta de seis etapas (figura 1), adecuadas a las características de este estudio a partir de un trabajo previo (1).

Una vez establecidos el objeto (el MC SPPIC) y el objetivo del estudio (identificar los cambios introducidos en el entramado constitucional y legal, y la política económica y social de Cuba que inciden en el derecho al acceso a la salud), se identificaron los elementos a tomar en cuenta:

- Constitución de la República de Cuba, adoptada en 2019 (19)

- Actualización de los Lineamientos de la Política Económica y Social para el quinquenio 2016-2021, adoptados en 2017 (20)

- Leyes, decretos leyes y otras disposiciones legales relacionados con la propiedad intelectual, fundamentalmente la industrial, en el período entre 2015 y 2020.

Se realizó la búsqueda de documentos, ajustada al período de tiempo analizado, en la Gaceta Oficial de la República de Cuba y los sitios en Internet de la Oficina Cubana de la Propiedad Industrial (OCPI), los organismos de la Administración Central del Estado y del Grupo de las Industrias Biotecnológica y Farmacéutica BioCubaFarma. Se extrajo la información de interés relacionada con la propiedad industrial, la salud, y la industria biotecnológica y farmacéutica cubana. La información recuperada responde a la implantación de la Política del Sistema de Propiedad Industrial, aprobada en Cuba en el año 2014 (9-11).

CUADRO 1. Principios de la Política del Sistema de Propiedad Industrial y del Sistema de Propiedad Industriala

\section{Política del Sistema de Propiedad Industrial}

Integrar la política de propiedad industrial a las políticas públicas y el desarrollo nacional.

Institucionalizar la propiedad industrial en los órganos y organismos de la Administración Central del Estado y organizaciones superiores de desarrollo empresarial para crear capacidades y competencias en la materia y garantizar la protección, la gestión, la comercialización y la defensa de los derechos de propiedad industrial en Cuba y en los mercados de interés.

Observar que los acuerdos internacionales que el país suscriba y las normas jurídicas internas no incluyan disposiciones que rebasen los estándares mínimos de protección o socaven las flexibilidades comprendidas en el Acuerdo sobre los Aspectos de los Derechos de Propiedad Intelectual relacionados con el Comercio.

Garantizar la protección y la gestión de los derechos de propiedad industrial y de la información no divulgada.

Incluir la enseñanza de la propiedad industrial en las universidades y otros centros de educación superior, así como perfeccionar la enseñanza de posgrado.

Remunerar a los inventores y autores a partir de los beneficios que se obtengan por la explotación de las creaciones en el marco de una relación laboral.

Realizar la evaluación económica de los activos intangibles de propiedad industrial y contabilizar los montos por la comercialización.

\section{Sistema de Propiedad Industrial}

Salvaguardar el interés público para el desarrollo científico-tecnológico y socioeconómico. Permitir la adopción de medidas necesarias para proteger la salud pública y la nutrición de la población.

Contrarrestar el ejercicio abusivo de los derechos de propiedad industrial que se adquieran y las prácticas que limiten de manera injustificada el comercio 0 redunden en detrimento de las transferencias de tecnologías.

Observar que los acuerdos internacionales que el país suscriba y las normas jurídicas internas no incluyan disposiciones que rebasen los estándares mínimos de protección o socaven las flexibilidades comprendidas en el Acuerdo sobre los Aspectos de los Derechos de Propiedad Intelectual relacionados con el Comercio

Garantizar la protección, la gestión y la comercialización de los derechos de propiedad industrial y de la información no divulgada. 
FIGURA 1. Etapas de la metodología para la generación y gestión del conocimiento aplicada a la elaboración del mapa conceptual sobre salud pública y propiedad intelectual en Cuba, 2020

\begin{tabular}{|c|l|}
\hline Identificación de objeto y objetivo & $\begin{array}{l}\text { Objeto: Mapa conceptual sobre salud pública y propiedad } \\
\text { intelectual en Cuba } \\
\text { Objetivo: Identificar los cambios ocurridos en Cuba en su } \\
\text { entramado constitucional y legal, y la política económica y social }\end{array}$ \\
\hline \begin{tabular}{|c|}
\hline $\begin{array}{c}\text { Identificación de elementos a } \\
\text { actualizar }\end{array}$ \\
\cline { 1 - 2 }
\end{tabular} & $\begin{array}{l}\text { Componentes del entramado constitucional, legal y } \\
\text { socioeconómico que inciden en el acceso a la salud y sufrieron } \\
\text { cambios en el período analizado }\end{array}$ \\
\hline
\end{tabular}

\begin{tabular}{|c|l|}
\hline \multicolumn{1}{|c|}{\begin{tabular}{|l|} 
Identificación de la estrategia de \\
búsqueda
\end{tabular}} & $\begin{array}{l}\text { Estrategia de búsqueda: documentos jurídico-legales } \\
\text { Período analizado: 2015-2020 } \\
\text { Fuentes: Gaceta Oficial de la República de Cuba, sitios en Internet de la } \\
\text { OCPI, los organismos de la Administración Central del Estado y } \\
\text { BioCubaFarma }\end{array}$ \\
\hline Recobrar información & $\begin{array}{l}\text { Se recuperó la información sobre leyes, decretos leyes, resoluciones } \\
\text { la nueva constitución y los lineamientos económicos y sociales } \\
\text { emitidos después del año } 2014\end{array}$ \\
\hline
\end{tabular}

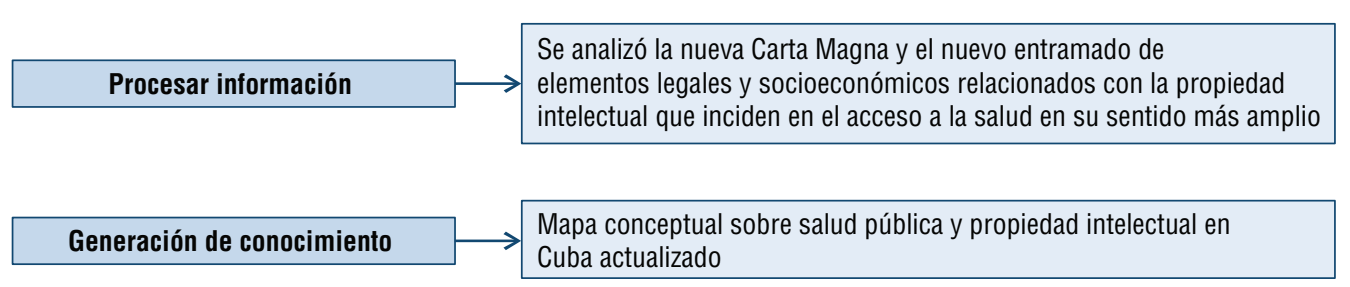

Fuente: Elaboración propia a partir de datos de las referencias 16-18

Nota: OCPI: Oficina Cubana de la Propiedad Industrial, BioCubaFarma: Grupo de las Industrias Biotecnológica y Farmacéutica BioCubaFarma.

\section{ANÁLISIS DE LOS DOCUMENTOS BÁSICOS DEL NUEVO MC SPPIC}

A partir de la información resultante (figura 2) se analizaron las bases documentales para llegar a una versión actualizada del MC SPPIC que reflejara la evolución de los derechos de los cubanos al acceso a la salud, así como lo regulado sobre propiedad intelectual y las proyecciones económicas y sociales del país.

\section{Constitución de la República de Cuba}

La nueva Carta Magna, aprobada tras una consulta popular en 2019 (19), refuerza en su Capítulo II los derechos de los ciudadanos cubanos en materia de salud, educación y propiedad intelectual y presenta un concepto más amplio y moderno de la salud pública, con un enfoque hacia lo social y el bienestar de la población. Lo anterior se establece en su Artículo 72, que plantea "la salud pública es un derecho de todas las personas y es responsabilidad del Estado garantizar el acceso, la gratuidad y la calidad de los servicios de atención, protección y recuperación. El Estado, para hacer efectivo este derecho, instituye un sistema de salud accesible a la población en todos sus niveles y desarrolla programas de prevención y educación, al que contribuyen la sociedad y las familias".

La nueva Constitución introduce cambios notables con relación a la Constitución de 1976 (21), la cual no hace alusión directa a la propiedad intelectual, ya sean los derechos de autor o la propiedad industrial (8). La Carta Magna actual difiere también de la anterior en lo que concierne a la actividad creadora, investigativa, de innovación y de derechos, con un reconocimiento explícito, por primera vez, de los derechos intelectuales de los creadores e investigadores. Esto quedó plasmado en el Artículo $32 \mathrm{f}$, que establece que "la actividad creadora e investigativa en la ciencia es libre, estimulando la investigación científica con un enfoque de desarrollo e innovación, priorizando la dirigida a solucionar los problemas que atañen al interés de la sociedad y al beneficio del pueblo" (19). También, en su artículo 62 se regulan los derechos intelectuales al disponer que "se reconocen a las personas los derechos derivados de la creación intelectual, conforme a la ley y a los tratados internacionales" y se establece que "los derechos adquiridos se ejercen por los creadores y titulares en correspondencia con la ley, en función de las políticas públicas".

\section{Lineamientos de la Política Económica y Social para el quinquenio 2016-2021}

En estos Lineamientos (20) se reafirma la prioridad conferida en Cuba a la salud pública y otras esferas relacionadas - como la educación, la ciencia y la industria médico-farmacéutica, entre otras-, ya refrendada en los lineamientos aprobados para el quinquenio 2011-2015 (22).

Uno de los nuevos lineamientos que potencian a la política industrial establece explícitamente como directriz: “Consolidar 
FIGURA 2. Principales cambios en el entramado constitucional y legal, y la política económica y social relacionados con la salud pública y la propiedad intelectual en Cuba, 2015-2020
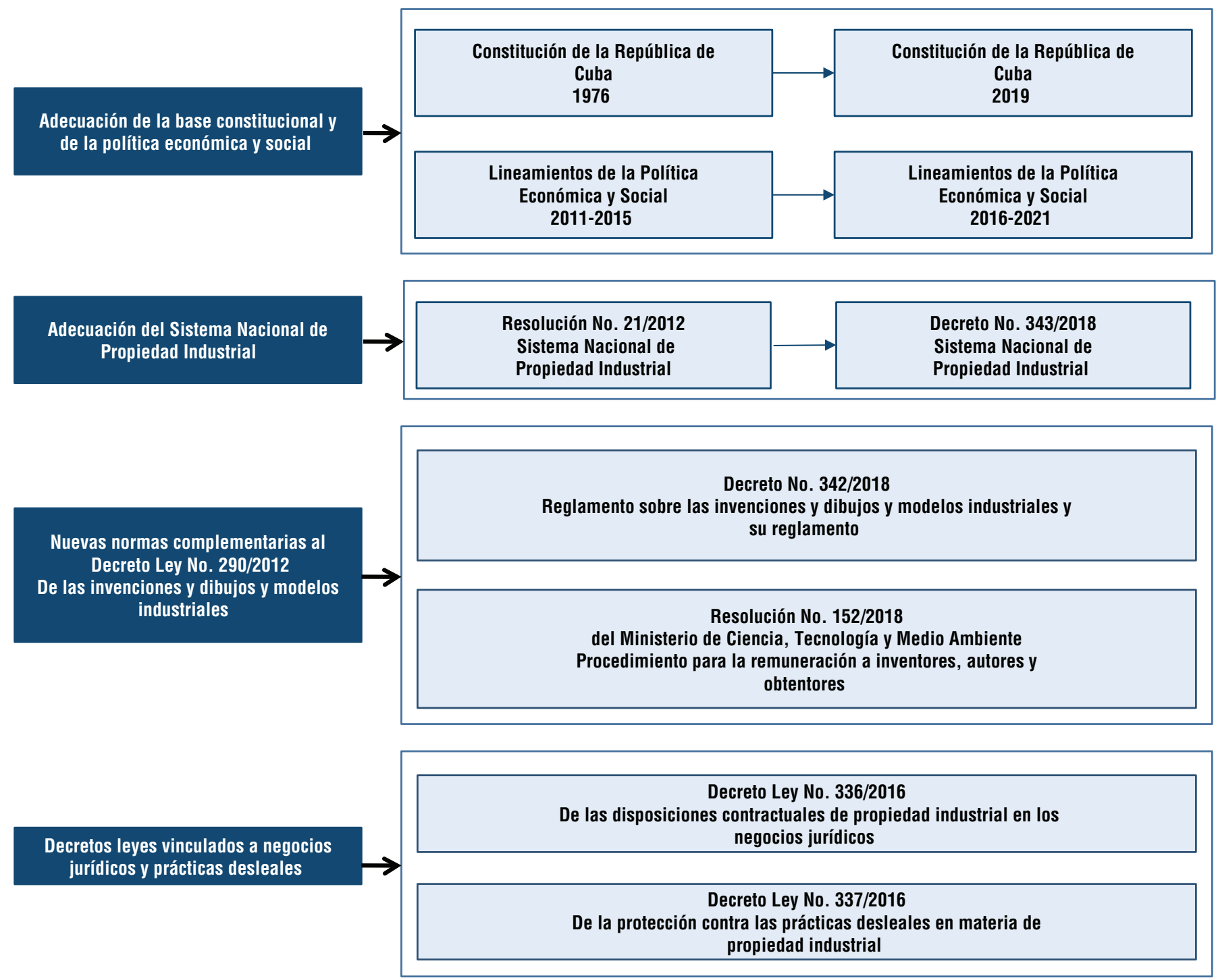

Fuente: Elaboración propia a partir de datos de las referencias 12 y 19-30.

la industria farmacéutica y biotecnológica como una de las actividades de mayor capacidad exportadora de la economía, diversificar productos y mercados e incorporar nuevos productos al mercado nacional para sustituir importaciones" (20).

Un hito en este sentido fue la creación en 2012 del grupo empresarial BioCubaFarma como organización superior de dirección subordinada directamente al Consejo de Ministros. BioCubaFarma está compuesto actualmente (mayo de 2020) por 34 empresas de ciclo cerrado (desde la investigación hasta la producción y la comercialización), algunas con más de 30 años de experiencia y que en conjunto ya cuentan con más de 1000 productos - de ellos 482 incluidos en el cuadro básico de medicamentos de Cuba (31)—, 765 registros sanitarios en más de 50 países y 2640 patentes (32). Este y otros desarrollos han contribuido a la consolidación de una industria biotecnológica y farmacéutica autóctona con tecnologías de última generación, institucionalizada mediante el Decreto No. 2/2020 (33), que establece y define la categoría de empresas de alta tecnología y su organización y funcionamiento. Este Decreto tiene entre sus bases el Artículo 21 de la Constitución, que establece que "el Estado promueve el avance de la ciencia, la tecnología y la innovación como elementos imprescindibles para el desarrollo económico y social", y continúa, "igualmente implementa formas de organización, financiamiento y gestión de la actividad científica; propicia la introducción sistemática y acelerada de sus resultados en los procesos productivos y de servicios, mediante el marco institucional y regulatorio correspondiente" (19).

Con relación a la política de ciencia, tecnología, innovación y medio ambiente, los Lineamientos de la Política Económica y Social orientan explícitamente "sostener y desarrollar los resultados alcanzados en el campo de la biotecnología, la producción médico-farmacéutica, las ciencias básicas, las ciencias naturales, la transferencia tecnológica industrial, la 
producción de equipos de tecnología avanzada, la nanotecnología y los servicios científicos y tecnológicos de alto valor agregado" e indican "fortalecer las capacidades de prospección y vigilancia tecnológica, así como la política de protección de la propiedad industrial en Cuba y en los principales mercados externos" (20).

\section{Disposiciones legales relacionadas con el Sistema de Propiedad Industrial}

Los cambios anteriores se complementan con la implantación de políticas públicas relacionadas con la ciencia, la educación, la salud y la propiedad intelectual -especialmente lo concerniente a las patentes de invención - a partir de disposiciones legales $(10,23)$.

En la última década, la legislación en materia de propiedad industrial se enriqueció con normas legales asociadas al sector de la salud y la industria vinculada a esta. Por ejemplo, el Decreto Ley No. 290/2012 sobre las invenciones y dibujos y modelos industriales (24) — que derogó algunos de los artículos del Decreto Ley No. 68/1983 sobre invenciones, descubrimientos científicos, modelos industriales, marcas y denominaciones de origen (25) - facilitó la presentación y modificación de solicitudes de patentes para productos farmacéuticos, entre otros.

Entre otras intervenciones públicas cabe destacar la aprobación de la Política del Sistema de Propiedad Industrial, diseñada como instru mento de gestión aplicable tanto a personas jurídicas como naturales. Esta Política permite consolidar el Sistema de Propiedad Industrial en el país y que se establezcan medidas de protección a la salud pública nacional, además de salvaguardar y estimular el desarrollo científico-tecnológico y económico-social del país $(9,10,31)$. Entre 2016 y 2018 se aprobaron decretos, decretos leyes y resoluciones que perfeccionan el ordenamiento jurídico existente en esta materia, entre los que se destacan por su trascendencia:

Decreto No. 342/2018. Este decreto (26) permite la instrumentación del Decreto Ley No. 290/2012 (24), que regula las invenciones y los dibujos y modelos industriales, y es de gran importancia para el sector de la salud por establecer el procedimiento de concesión de licencias obligatorias.

Resolución 152/2018. Emitida por el Ministerio de Ciencia, Tecnología y Medio Ambiente, esta resolución (28) establece el procedimiento para hacer efectivo el derecho de los autores, inventores y obtentores a participar de los beneficios que los titulares obtengan por la explotación de creaciones realizadas en el marco de una relación de trabajo. Esta Resolución es también una norma complementaria del Decreto Ley No. 290/2012 (24).

Las empresas de alta tecnología y otras instituciones biotecnológicas y farmacéuticas que cuentan con patentes se benefician con la implantación de este Reglamento porque permite el uso de las flexibilidades previstas en el Acuerdo sobre los Aspectos de los Derechos de Propiedad Intelectual relacionados con el Comercio - de gran importancia para la producción de medicamentos genéricos- y por la posibilidad de ofrecer beneficios económicos a los autores de invenciones comercializadas. Esto constituye una motivación y un estímulo más para el desarrollo de nuevos productos y tecnologías dirigidos a la salud.

Decreto No. 343/2018. Este decreto (12) sustituye a la Resolución 21/2002 (27). En él se establece que el Sistema de Propiedad Industrial está constituido por el conjunto de relaciones funcionales para la gestión de la propiedad industrial en la República de Cuba y en otros mercados de interés y se fundamenta en los principios de políticas, normas nacionales, y tratados y acuerdos internacionales suscritos por Cuba en la materia. Este Sistema está integrado por la OCPI, como institución rectora, otros órganos y organismos de la Administración Central del Estado, las organizaciones superiores de dirección empresarial y otras empresas y entidades nacionales.

Decreto-Ley No. 336/2016. Establece las normas relativas a las disposiciones contractuales de propiedad industrial que se deben incluir en los negocios jurídicos para la adquisición de tecnologías y en los acuerdos de colaboración económica y científico-técnica (29). En él se identifican las disposiciones contractuales restrictivas que impiden, limitan o distorsionan abusiva o injustificadamente el desempeño industrial y comercial. Con este decreto ley se da respuesta a una de las recomendaciones de la actualización del modelo económico y social cubano (20).

Decreto Ley No. 337/2016. Este decreto ley (30) establece las normas para la protección contra las prácticas desleales en materia de propiedad industrial, que contempla la información no divulgada legítimamente bajo el control de personas naturales y jurídicas, y determinados datos de prueba depositados ante las autoridades reguladoras que aprueban la comercialización de productos farmacéuticos u otros. En él se establece que la protección contra las prácticas desleales debe estar en correspondencia con las características y las necesidades del mercado nacional, y los objetivos económicos y sociales de desarrollo del país, así como con las políticas públicas. Su entrada en vigor en 2018 dio un gran respaldo al desarrollo de la industria biotecnológica y farmacéutica.

\section{ANÁLISIS DE LOS ACTORES EN EL NUEVO MC SPPIC}

Los cambios que han tenido lugar en el período 2015-2020 (figura 2) han potenciado tanto el Sistema Nacional de Salud como el Sistema de Propiedad Industrial gracias a la convergencia de las políticas nacionales y sectoriales de propiedad industrial que inciden en el derecho al acceso a la salud.

La OCPI se mantiene a cargo de la administración de la propiedad industrial, incluida la relacionada con la salud. A partir de esos cambios se incorpora al MC SPPIC el Centro para el Control Estatal de Medicamentos, Equipos y Dispositivos Médicos, que protege contra todo uso comercial desleal de los datos de prueba $u$ otros datos no divulgados que se requieran para autorizar la comercialización de productos farmacéuticos que utilizan nuevas entidades químicas. Por su parte, las políticas sectoriales de propiedad industrial relacionadas con la salud están vinculadas con un considerable grupo de organismos centrales del Estado —el Ministerio de Salud Pública; el Ministerio de Ciencia, Tecnología y Medio Ambiente; el Ministerio de Educación Superior; el Ministerio de Comercio Exterior y la Inversión Extranjera; y el Ministerio de Relaciones Exteriores, entre otros-, además de BioCubaFarma y otras dependencias de subordinación nacional. En la observancia de los derechos de propiedad industrial participan también la Aduana General de la República y el sistema de tribunales del país por su función en la vigilancia y en hacer cumplir lo establecido (figura 3). Los cambios introducidos han fortalecido la interrelación de los actores estatales y sociales vinculados con la salud pública y la propiedad intelectual. 


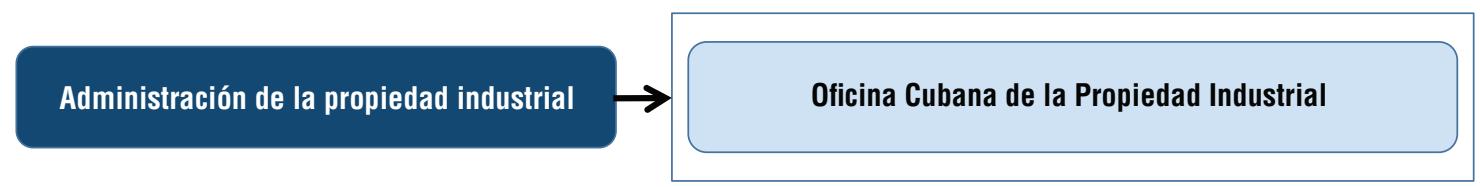

Proteceión contra el uso desleal de datos de prueba u otros datos no divulgados

Desarrollo de las políticas sectoriales de propiedad industrial relacionadas con la salud (entre otros)
Centro para el Control Estatal de Medicamentos, Equipos y Dispositivos Médicos

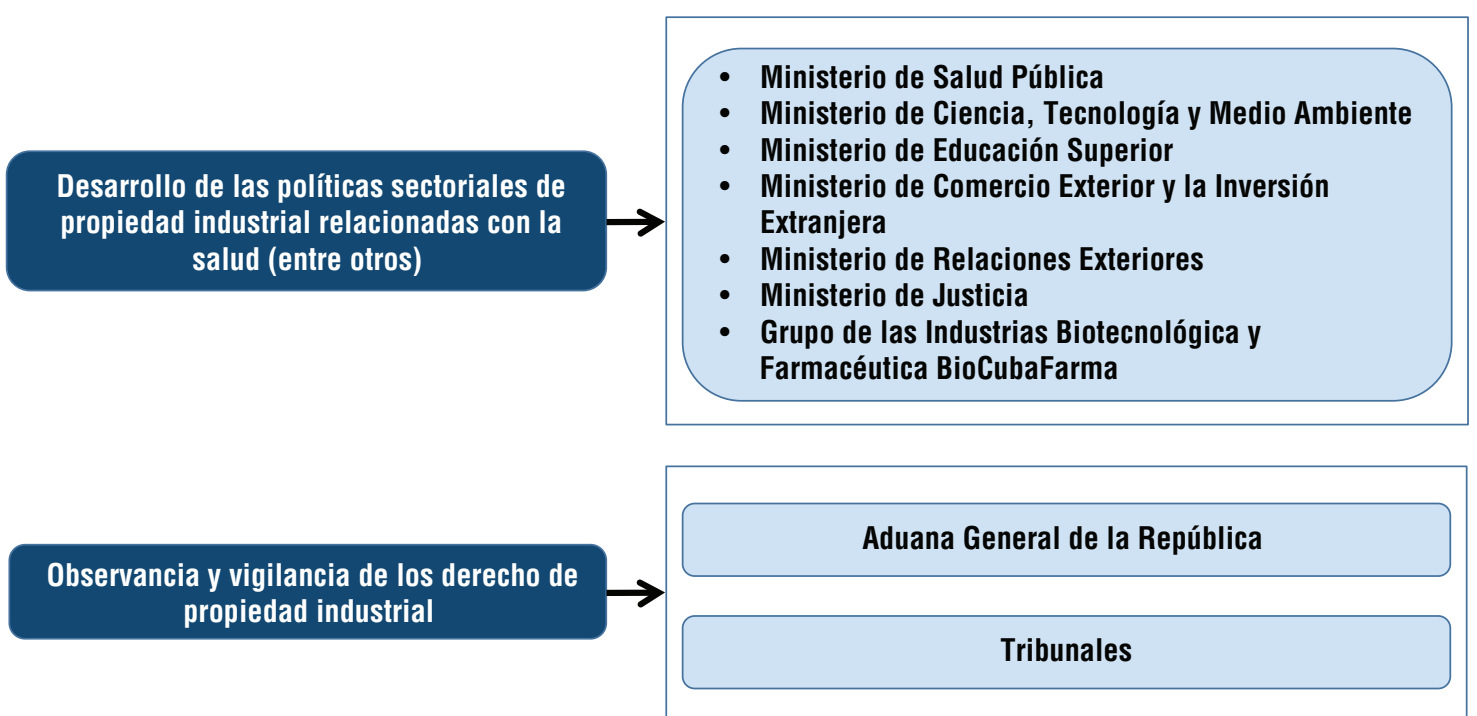

Fuente: Elaboración propia a partir de datos de las referencias $9,12,19,20,23,26,29,30$ y 33 .

El pleno acceso a las tecnologías sanitarias es un tema en el cual inciden diferentes aristas y actores y para lograrlo debe existir, en primer lugar, una clara voluntad política en todos los niveles de gobierno del país y, por consiguiente, un andamiaje jurídico y estructural que permita que se materialice. Esto hace que se deban tomar en consideración factores externos e internos que, de una forma $u$ otra, inciden positiva o negativamente en el acceso a esas tecnologías.

En Cuba, esa voluntad política se ha mantenido de forma coherente durante seis décadas y lo aprobado en la nueva Constitución - en particular lo que respecta al derecho a la salud y a la propiedad industrial- lo demuestra, y las políticas aprobadas en materia de salud, ciencia, tecnología y propiedad industrial lo reafirman

La actualización de los enunciados de los Lineamientos de la Política Económica y Social relacionados con estas esferas es otra muestra de la voluntad política del Estado cubano, ya que en ellos se refuerza el derecho a la salud, se incentiva la investigación-desarrollo en la industria y se reafirma el concepto de que sus resultados deben dirigirse a satisfacer las necesidades de la población en materia de salud.

Los ministerios de Educación Superior y de Salud Pública desempeñan un papel fundamental en la formación de capital humano en todo lo relacionado con la salud pública. En el año 2018 se aprobaron los cursos de ciclo corto como nueva modalidad de la educación superior, siempre bajo la dirección y tutela de esos ministerios (34). Los centros del sistema de educación superior — tanto las universidades como las instituciones científicas regidas por ese ministerio- participan en investigaciones dirigidas al desarrollo de tecnologías sanitarias que nutren a la industria biotecnológica y farmacéutica y al Sistema Nacional de Salud. Lo anterior se ha materializado, por ejemplo, en el creciente número de proyectos conjuntos de BioCubaFarma con la Universidad de La Habana y con la Universidad de Ciencias Informáticas, y en la creación de nuevos laboratorios en la Facultad de Química y el Instituto de Farmacia y Alimentos, ambos de la Universidad de La Habana, entre otros (35-37).

El Ministerio de Comercio Exterior y la Inversión Extranjera es otro de los organismos que integra este mapa y forma parte esencial de la contextualización del Sistema de Propiedad Industrial. Este ministerio (solo o conjuntamente con otros organismos nacionales) es fundamental por su participación en acuerdos gubernamentales, regionales y de cooperación de los que Cuba es signataria, y por coordinar las negociaciones con organismos internacionales como la Organización Mundial de la Propiedad Intelectual, la Organización Mundial del Comercio, la OMS, la OPS, la Organización de las Naciones Unidas para la Agricultura y la Alimentación, y la Conferencia de las Naciones Unidas sobre Comercio y Desarrollo. 
La Aduana General de la República y los tribunales son también actores fundamentales por su responsabilidad en la vigilancia y en hacer cumplir lo regulado sobre derechos de propiedad industrial.

En el período estudiado, BioCubaFarma ha continuado desarrollando novedosas tecnologías y productos, con una estrategia sistemática de protección de los mismos en Cuba y en el extranjero, lo que le ha permitido ampliar su valioso portafolio de patentes y marcas registradas en todo el mundo. Esto es una expresión del impacto tecnológico y las posibilidades comerciales de los resultados obtenidos por ese grupo de instituciones. A modo de ejemplo, se debe mencionar el Heberprot- $\mathrm{P}^{\circledR}$, fármaco que permite reducir la necesidad de amputaciones en personas con úlceras de pie diabético; es un producto único y exclusivo, del cual se poseen los derechos de propiedad industrial (patentes) registrados en más de 30 países, incluidos los Estados Unidos de América (32, 38).

\section{NUEVO MC SPPIC}

En el MC SPPIC actualizado (figura 4) se toman en cuenta y visualizan los cambios registrados en el entramado constitucional y legal, y la política económica y social ocurridos en Cuba en el período de 2015 a 2020 que inciden en el acceso a las tecnologías sanitarias y, por ende, a los servicios de salud.

Esta actualización refleja el desarrollo continuado de políticas científicas e industriales dirigidas a responder a las necesidades de la población y a garantizar que los resultados de las investigaciones sean utilizados, en primer lugar, en beneficio de la sociedad.

El nuevo MC SPPIC muestra, además, cómo la evolución política en Cuba se afianza coherentemente en el conjunto de leyes y normas que rigen la protección de la propiedad intelectual y que guían el desarrollo y el funcionamiento de la investigación biomédica y la industria farmacéutica, de titularidad pública, a partir de lo establecido en la Constitución de la República y los Lineamientos de la Política Económica y Social del país.

Destaca igualmente que la protección y la promoción del derecho a la salud de todos los cubanos y cubanas siguen siendo prioridades para el Estado y guían la articulación de los diversos estamentos legales, científicos y profesionales, incluida la gestión de los derechos de propiedad industrial y la promoción de la investigación biomédica, a fin de responder a las necesidades de las mayorías en el ámbito sanitario.

\section{Mapa Conceptual en Salud Pública y Propiedad Intelectual, Cuba 2020}

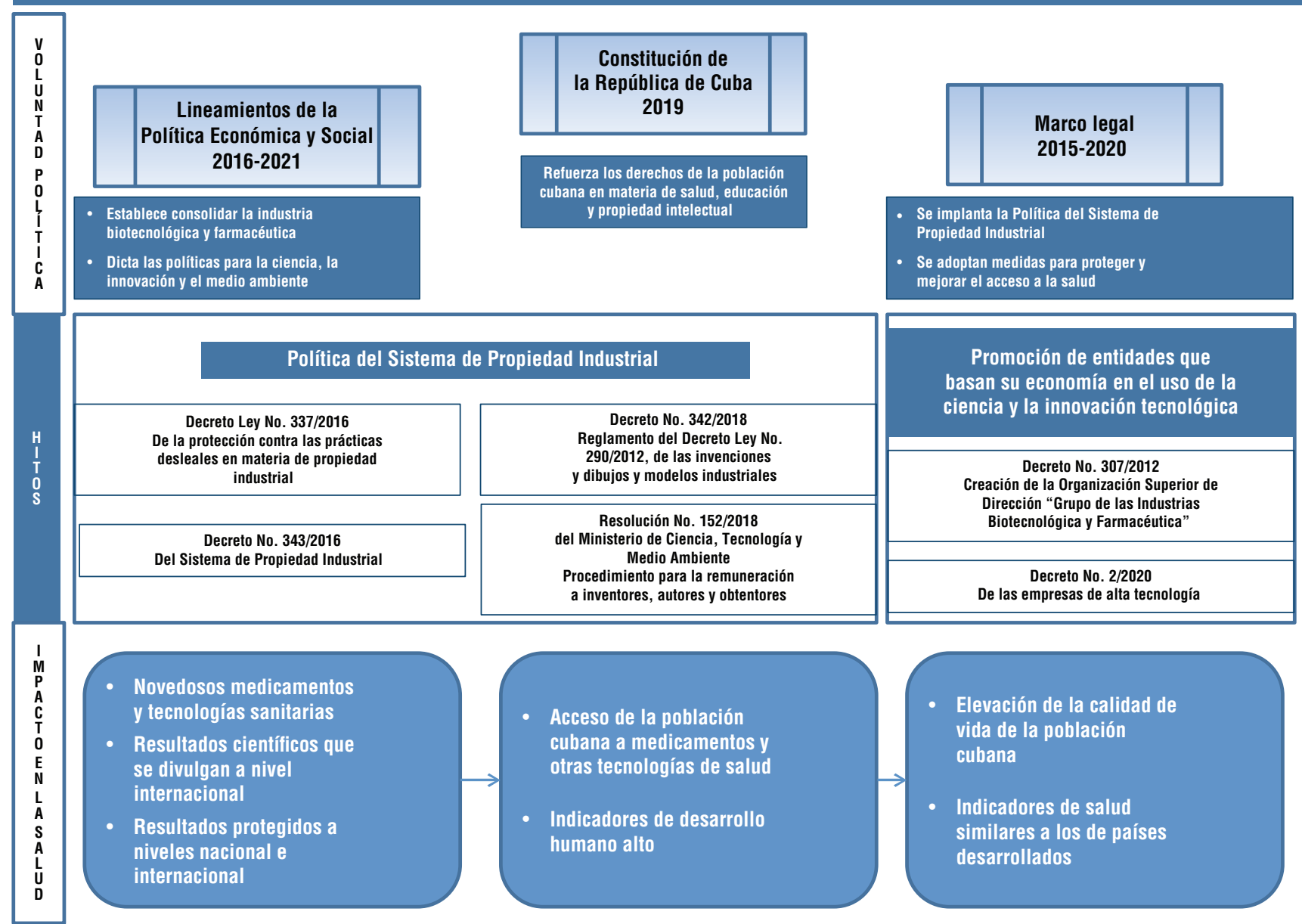

Fuente: Elaboración propia a partir de datos de las referencias 9, 12, 19, 20, 23, 26, 29, 30, 32, 33 y 39. 
La Política sobre el Sistema de Propiedad Industrial aprobada se fortaleció con legislaciones relacionadas con la protección de la propiedad industrial, en especial las que inciden en la protección de los resultados obtenidos por la industria biotecnológica y farmacéutica cubana, la cual continúa desarrollando novedosos medicamentos y tecnologías sanitarias. La implementación de esta Política y su entramado legal incide positivamente en el desarrollo de nuevos productos y tecnologías para la industria biotecnológica y farmacéutica nacional.

\section{CONCLUSIONES Y RECOMENDACIONES}

Los cambios ocurridos en el entramado constitucional y legal, y la política económica y social de Cuba entre los años 2015 y 2020, que quedan reflejados en el MC SPPIC actualizado, contribuyen a una mayor interacción y sinergia entre los diversos actores que inciden en el acceso a la salud - en su sentido más amplio- de la población cubana. Se debe continuar estudiando el impacto de la implementación de los cambios señalados y de las diferentes políticas trazadas a todos los niveles en el país para ampliar aun más el acceso a la salud de la población.

Se recomienda reevaluar el impacto en el acceso a la salud de los cambios que se produzcan en los próximos años a fin de mantener actualizado el MC SPPIC.

Contribución de los autores. BGM y JLDF concibieron el estudio original y recolectaron los datos. Todos los autores participaron en el análisis de los datos, la interpretación de los resultados, y la redacción y revisión del manuscrito. Todos los autores revisaron y aprobaron la versión final.

\section{Conflictos de intereses. Ninguno.}

Declaración. Las opiniones expresadas en este manuscrito son responsabilidad del autor y no reflejan necesariamente los criterios ni la política de European Projects Health Action International, la RPSP/PAJPH y/o de la OPS.

\section{REFERENCIAS}

1. García BM, Di Fabio JL, Vidal J, Fitzgerald J, Silva AP. Salud pública y propiedad intelectual en Cuba: mapa conceptual. Rev Panam Salud Publica. 2015;38(5):355-61.

2. Organización Mundial de la Salud. 61. a Asamblea Mundial de la Salud. Estrategia mundial y plan de acción sobre salud pública, innovación y propiedad intelectual. Ginebra: OMS; 2008 (Resolución WHA61.21) [citado el 19 de marzo de 2020]. Disponible en: https://apps.who.int/iris/handle/10665/26290

3. Organización Panamericana de la Salud. $48 .{ }^{\circ}$ Consejo Directivo, $60 .{ }^{a}$ Sesión del Comité Regional. Salud pública, innovación y propiedad intelectual: una perspectiva regional. Washington, DC.: OPS; 2008 (Resolución CD48.R15) [citado el 19 de marzo de 2020]. Disponible en: http://www1.paho.org/spanish/gov/cd/cd48.r15-s.pdf

4. Organización Mundial de la Salud. Acceso a medicamentos y vacunas. Informe del Director General. Ginebra: OMS; 2019 (Documento A72/17) [citado el 19 de marzo de 2020]. Disponible en: http:// apps.who.int/gb/s/s_wha72.html

5. García BM, Silva AP, de Rodríguez JM. Mapa conceptual en salud y propiedad intelectual en Centroamérica y la República Dominicana. Rev Panam Salud Publica. 2019;43:e4 [citado el 2 de marzo de 2020]. Disponible en: https:/ / doi.org/10.26633/RPSP.2019.4

6. Leyns C, Maeseneer J, Willems S. Using concept mapping to identify policy options and interventions towards people centered health care services: a multi-stakeholder perspective. Int J Equity Health. 2018;17:177.

7. Tromichi W, Kanet M. Concept mapping: an introduction to structured conceptualization in health care. Int J Qual Health Care. 2005;17(3):187-91 [citado el 25 de marzo de 2020]. Disponible en: https:/ / www.ncbi.nlm.nih.gov/pubmed/15872026

8. Cándano M, Moreno M. Propiedad intelectual en Cuba: una mirada crítica a su reconocimiento constitucional. Rev Chil Derecho Tecnol. 2019;8(1):133-65 [citado el 2 de marzo de 2020]. Disponible en: http://dx.doi.org/10.5354/0719-2584.2019.51115

9. Sánchez MA. Sistema de propiedad industrial. Actualización sobre las normas jurídicas que implementan la política del sistema de propiedad industrial [videoconferencia]. Capacitación a las Universidades. La Habana: Oficina Cubana de la Propiedad Industrial; 2019 [citado el 1 de marzo de 2020]. Disponible en: http://www .ocpi.cu/sites/default/files/Eventos/Conferencias/sistema_0.pdf

10. Establecen normas jurídicas para el Sistema de Propiedad Industrial cubano. Cubadebate. 2018 agosto 10 [citado el 2 de marzo de 2020]. Disponible en: http://www.cubadebate.cu/noticias/2018 /08/10/establecen-normas-juridicas-para-el-sistema-de-propiedad -industrial-cubano/
11. Oficina Cubana de la Propiedad Industrial. Nuevas normas jurídicas de propiedad industrial. La Habana: OCPI; 2018 [citado el 7 de marzo de 2020]. Disponible en: http:/ / www.ocpi.cu/node/1825

12. República de Cuba, Consejo de Ministros. Decreto No. 343/2018. Del Sistema de Propiedad Industrial. Gac Of Repub Cuba. 2018;(40 Extraordinaria):783-94 [citado el 8 de marzo de 2020]. Disponible en: http://www.ocpi.cu/sites/default/files/legislacion/decre to343.pdf

13. Cadalso Y, Becerra MJ, Pulido A, Caballero I, Albojaire M. Regularidades de la gestión del capital humano en empresas de alta tecnología del sector biotecnológico farmacéutico cubano. COFIN. 2019;13(1):e08 [citado el 5 de marzo de 2020]. Disponible en: http:/ / scielo.sld.cu/pdf/cofin/v13n1/2073-6061-cofin-13-01-e08.pdf

14. Lage A. La osadía de la ciencia. La Habana: Editorial Academia; 2018.

15. Lage A. Science and challenges for Cuban public health in the 21..$^{\text {st }}$ century. MEDICC Rev. 2019;21(4):7-14 [citado el 8 de marzo de 2020]. Disponible en: http://mediccreview.org/wp-content /uploads/2019/09/MR-September 2019-Lage-Science-Challenges. pdf

16. García BM. Gestión y generación de conocimientos a partir de la información de patentes: metodología [tesis de maestría]. La Habana: Oficina Cubana de Propiedad Intelectual; 2012 [citado el 5 de marzo de 2020]. Disponible en: https:/ /www.researchgate.net /publication/323014459

17. García B, Delgado M. Sistematización y diversificación de la metodología para la generación de conocimientos. An Acad Cienc Cuba. 2019;9(3):1-4 [citado el 19 de marzo de 2020]. Disponible en: http:/ / www.revistaccuba.cu/index.php/revacc/article/view/698/716

18. García B, Delgado M, Infante M. Metodología para la generación y gestión del conocimiento para proyectos de I+D+i vista desde sus factores críticos. Rev Cubana Inf Cienc Salud. 2014;25(3):285-302.

19. Constitución de la República de Cuba. Cubadebate. 2019 abril 10 [citado el 16 de marzo de 2020]. Disponible en: http://media .cubadebate.cu/wp-content/uploads/2019/01/Constitucion-Cuba -2019.pdf

20. Partido Comunista de Cuba. Actualización de los Lineamientos de la Política Económica y Social del Partido y la Revolución para el período 2016-2021. La Habana: Editora Política; 2016 [citado el 19 de marzo de 2020]. Disponible en: http://www.granma.cu/file/pdf /gaceta/01Folleto.Lineamientos-4.pdf

21. Constitución de la República de Cuba. La Habana: Ministerio de Justicia; 1976 [citado el 6 de marzo de 2020]. Disponible en: http:/ / www.cuba.cu/gobierno/cuba.htm 
22. Partido Comunista de Cuba. Lineamientos de la Política Económica y Social del Partido y la Revolución. La Habana: Cubadebate; 2011 [citado el 6 de febrero de 2020]. Disponible en: http://www .cubadebate.cu/noticias/2011/05/09/descargue-en-cubadebate -los-lineamientos-de-la-politica-economica-y-social-pdf/

23. Oficina Cubana de la Propiedad Industrial. Nuevas normas sobre el Sistema de Propiedad Industrial. La Habana: OCPI; 2016 [citado el 6 de marzo de 2020]. Disponible en: http://www.ocpi.cu/node $/ 1746$

24. República de Cuba, Consejo de Estado. Decreto Ley No. 290/2012. De las invenciones, dibujos y modelos industriales. Gac Of Repub Cuba. 2012;(24 Extraordinaria):783-94 [citado el 2 de marzo de 2020]. Disponible en: http://www.ocpi.cu/sites/default/files/legislacion /decreto290modificado-0.pdf

25. República de Cuba, Consejo de Estado. Decreto Ley No. 68/1983. De invenciones, descubrimientos científicos, modelos industriales, marcas y denominaciones de origen. Gac Of Repub Cuba. 1983;(10 Extraordinaria):81-96 [citado el 8 de marzo de 2020]. Disponible en: http://www.ocpi.cu/sites/default/files/legislacion/LEY68.pdf

26. República de Cuba, Consejo de Ministros. Decreto No. 342/2018. Reglamento del Decreto Ley No. 290/2012 de las invenciones y dibujos y modelos industriales. Gac Of Repub Cuba. 2018;(40 Extraordinaria):761-83 [citado el 8 de marzo de 2020]. Disponible en: http://www.ocpi.cu/sites/default/files/legislacion/342.pdf

27. República de Cuba, Ministerio de Ciencia, Tecnología y Medio Ambiente. Resolución 21/2002. Sobre el Sistema Nacional de la Propiedad Industrial. Gac Of Repub Cuba. 2003;(21):24-32 [citado el 8 de marzo de 2020]. Disponible en: http://www.ocpi.cu/doc/legis /RES2102.pdf

28. República de Cuba, Ministerio de Ciencia, Tecnología y Medio Ambiente. Resolución 152/2018. Procedimiento para la remuneración a inventores, autores y obtentores. Gac Of Repub Cuba. 2018:(40 Extraordinaria):805-6 [citado el 8 de marzo de 2020]. Disponible en: http://www.ocpi.cu/sites/default/files/legislacion/re solucion152.pdf

29. República de Cuba, Consejo de Estado. Decreto Ley No. 336/2016. De las disposiciones contractuales de propiedad industrial en los negocios jurídicos. Gac Of Repub Cuba. 2018;(40 Extraordinaria):745-56 [citado el 8 de marzo de 2020]. Disponible en: http:// www.ocpi.cu/sites/default/files/legislacion/336.pdf

30. República de Cuba, Consejo de Estado. Decreto Ley No. 337/2016. De la protección contra las prácticas desleales en materia de propiedad industrial. Gac Of Repub Cuba. 2018;(40 Extraordinaria):757-61 [citado el 8 de marzo de 2020]. Disponible en: http:/ / www.ocpi.cu /sites/default/ files/legislacion/337.pdf

31. República de Cuba, Ministerio de Salud Pública. Cuadro básico de medicamentos. La Habana: Editorial Ciencias Médicas; 2018 [citado el 26 de abril de 2020]. Disponible en: http://temas.sld.cu /medicamentosterapeutica/2018/01/26/modificaciones-al-cuadro -basico-de-medicamentos-de-cuba-para-el-2018/

32. BioCubaFarma. Grupo de las Industrias Biotecnológica y Farmacéutica de Cuba. La Habana: BioCubaFarma; 2020 [citado el 26 de abril de 2020] Disponible en: https:/ / www.biocubafarma.cu/\#seccion1

33. República de Cuba, Consejo de Ministros. Decreto No. 2/2020. De las empresas de alta tecnología. Gac Of Repub Cuba. 2020;(16):43341 [citado el 26 de marzo de 2020]. Disponible en: https://www .gacetaoficial.gob.cu/sites/default/files/goc-2020-o16.pdf

34. República de Cuba, Ministerio de Educación Superior. Nivel de educación superior de ciclo corto. La Habana: MES; 2018 [citado el 8 de marzo de 2020]. Disponible en: https://www.mes.gob.cu/es/nivel -de-educacion-superior-de-ciclo-corto

35. De Armas Padrino I. Suscriben convenio Universidad de La Habana y BioCubaFarma. Agencia Cubana de Noticias. 2018 febrero 14 [citado el 3 de marzo de 2020]. Disponible en: http:// www.acn.cu/salud/32078-suscriben-convenio-universidad-dela--ybiocubafarma

36. Riverón A. Nuevas perspectivas en balance de trabajo entre la UCI y BioCubaFarma. La Habana: Universidad de Ciencias Informáticas, 2018 [citado el 3 de marzo de 2020]. Disponible en https://www .uci.cu/universidad/noticias/nuevas-perspectivas-en-balance-de -trabajo-entre-la-uci-y-biocubafarma

37. Académicos belgas y cubanos comparten experiencias sobre empresas de transferencia tecnológica de las universidades. La Habana: Delegación de la Unión Europea en Cuba; 2019 [citado el 10 de marzo de 2020]. Disponible en: https://eeas.europa.eu/delegations /cuba / 68160/acad\%C3\%A9micos-belgas-y-cubanos-comparten -experiencias-sobre-empresas-de-transferencia-tecnol\%C3\%B3gica_id

38. Fabelo A, Figueroa A, Valdés C, Pérez D, Álvarez A. Evolución de las úlceras de pie diabético con el tratamiento mixto de Heberprot-P ${ }^{\circledR}$ y ozonoterapia. Rev Cubana Angiol Cir Vasc. 2019;20(1) [citado el 10 de marzo de 2020]. Disponible en: https:/ / pesquisa.bvsalud.org /portal/resource/pt/biblio-991042

39. República de Cuba, Consejo de Ministros. Decreto No. 307/2012. Creación de la Organización Superior de Dirección Grupo de las Industrias Biotecnológica y Farmacéutica. Gac Of Repub Cuba 2012;(52 Extraordinaria):243-5 [citado el 10 de marzo de 2020]. Disponible en: https://www.gacetaoficial.gob.cu/es/decreto-307-de-2 012-de-consejo-de-ministros

Manuscrito recibido el 15 de octubre del 2019. Aceptado para publicación, tras revisión, el 22 de abril del 2020. 


\section{Conceptual map of public health and intellectual property in Cuba: 2020 update}

SUMMARY

Keywords Intellectual property; legislation as topic; drug industry; right to health; Cuba.

This study identifies and describes the changes introduced in Cuba's constitutional and legal framework, and the country's economic and social policy between 2015 and 2020, in terms of the effects on access to health. The conceptual map of public health and intellectual property in Cuba was also updated. A document search for the time period of the study was conducted in the Official Gazette of the Republic of Cuba and on the webpages of the Cuban Office of Industrial Property, Cuban government agencies, and the Cuban Biotechnology and Pharmaceutical Industries group (BioCubaFarma). The updated conceptual map reflects amendments to the new Constitution of the Republic of Cuba adopted in 2019, updated guidelines on economic and social policy for the quinquennium 2016-2021, and laws, decrees, and legal provisions adopted in the period 2015-2020 in relation to intellectual property. The approved policy on the industrial property system was strengthened with specific legislation to protect industrial property, especially for the protection of the results obtained by the Cuban biotechnology and pharmaceutical industry. The changes reflected in the updated conceptual map favor interactions and synergy among the various actors that affect the Cuban population's access to health in the broadest sense.

\section{Mapa conceitual sobre saúde pública e propriedade intelectual em Cuba: atualização de 2020}

RESUMO

Neste estudo, procuramos identificar e descrever as mudanças introduzidas no quadro constitucional e legal e na política econômica e social de Cuba entre 2015 e 2020 que afetam o acesso à saúde, bem como atualizar o mapa conceitual sobre saúde pública e propriedade intelectual em Cuba (MC SPPIC). Realizamos uma busca em documentos, ajustada ao período analisado, no Diário Oficial da República de Cuba e nos sites do Escritório Cubano de Propriedade Industrial, dos órgãos da Administração Central do Estado e do grupo da indústria biotecnológica e farmacêutica BioCubaFarma. O MC SPPIC atualizado reflete as mudanças na nova Constituição da República de Cuba adotada em 2019, a atualização das Diretrizes de Política Econômica e Social para o quinquênio 2016-2021 e as leis, decretos-lei e outras disposições legais relacionadas à propriedade intelectual no período 2015-2020. A Política sobre o Sistema de Propriedade Industrial, adotada previamente, foi fortalecida com legislação específica para a proteção da propriedade industrial, especialmente no que diz respeito à proteção dos resultados obtidos pela indústria biotecnológica e farmacêutica cubana. As mudanças refletidas no MC SPPIC atualizado favorecem a interação e sinergia entre os diversos atores que influenciam o acesso à saúde — em seu sentido mais amplo — por parte da população cubana.

Palavras-chave Propriedade intelectual; legislação como assunto; indústria farmacêutica; direito à saúde; Cuba. 